\title{
Quantum distillation in QCD
}

\author{
Takuya Kanazawa $\odot^{1}$ and Mithat Ünsal ${ }^{2}$ \\ ${ }^{1}$ Research and Development Group, Hitachi, Ltd., Kokubunji, Tokyo 185-8601, Japan \\ ${ }^{2}$ Department of Physics, North Carolina State University, \\ Raleigh, North Carolina 27695, USA
}

(Received 26 September 2019; accepted 27 July 2020; published 12 August 2020)

\begin{abstract}
We propose a grading protocol that assigns global symmetry associated phases to states in the Hilbert space. Without modifying the Hilbert space, this changes the state sum, a process that we call quantum distillation. We describe the image of quantum distillation in terms of (nondynamical) flavor holonomy dependence of (dynamical) gauge-holonomy potentials, in QCD with $N_{f}=N_{c}$ fundamental and one massive adjoint fermion on $\mathbb{R}^{3} \times S^{1}$. The compactified theory possesses an exact zero-form color-flavor center symmetry for a special choice of flavor holonomy (under which Polyakov loop is charged), despite the absence of one-form center symmetry. We prove that the color-flavor center symmetry is stable at small $\beta$. This is the opposite of the high-temperature limit of thermal theory and a dramatic manifestation of quantum distillation. We show chiral symmetry breaking at small $S^{1}$ and that the vacuum structure of the theory on $\mathbb{R}^{4}$ and $\mathbb{R}^{3} \times S^{1}$ are controlled by the same mixed 't Hooft anomaly condition.
\end{abstract}

DOI: 10.1103/PhysRevD.102.034013

\section{INTRODUCTION}

Consider a generic quantum field theory (QFT) on a $d$ dimensional space-manifold $\mathcal{M}$. The dimension of the Hilbert space of such a theory scales exponentially with the volume, $e^{\operatorname{Vol}(\mathcal{M})}$. It is a monstrous structure, and for different purposes, one cares very little with the detailed knowledge of the full space. For low energy physics, one cares solely about ground states and low lying states. In the theory of phase transitions, the interest is in the growth of the density of states $\rho(E)$ and high energy states become crucial.

Consider a thermal partition function associated with an asymptotically free or superrenormalizable QFT, $\mathcal{Z}(\beta)=\operatorname{tr}\left[e^{-\beta H}\right]$, where $\beta$ is inverse temperature. In the $\beta \rightarrow \infty$ limit, this receives dominant contributions from ground states and low lying states. However, in this limit, these QFTs are often strongly coupled and not amenable to analytic treatment. As $\beta \rightarrow 0$, they may become weakly coupled, but the state sum receives contributions from every state on the same footing. It is an utterly contaminated quantity. In this regime, it is impossible to isolate and understand the role of ground states and low lying states.

Published by the American Physical Society under the terms of the Creative Commons Attribution 4.0 International license. Further distribution of this work must maintain attribution to the author(s) and the published article's title, journal citation, and DOI. Funded by SCOAP ${ }^{3}$.
Relatedly, the theory often moves to a different phase at some $\beta<\beta_{c}$ due to phase transitions associated with singularities of partition function $\mathcal{Z}(\beta)$.

On the other hand, despite the fact that the full Hilbert spaces of supersymmetric theories are also equally complicated, one can often succeed in constructing graded state sums, e.g., supersymmetric Witten index [1], which receives contributions only from ground states, $\mathcal{Z}(\beta)=$ $\operatorname{tr}\left[e^{-\beta H}(-1)^{F}\right]$. This is a Bose-Fermi paired graded state sum, in which positive energy states $E>0$ cancel pairwise thanks to supersymmetry (assuming that the spectrum is discrete).

In this work, we are after something ambitious. We would like to construct a graded state sum in certain fourdimensional QCD-like theories, such that the cancellation among states can prevent all phase transitions as one moves from large $\beta$ to small $\beta$. We would like to achieve this without changing the theory, its Hilbert space, and without adding center-stabilizing double-trace deformations $[2,3]$.

The goal is to construct a graded state sum which can avoid all singularities in $\mathcal{Z}(\beta)$. One may think that this is impossible in a nontrivial nonsupersymmetric theory due to lack of supersymmetry. However, we will argue otherwise in interesting theories, including $N_{f}=N_{c}$ QCD with an extra heavy degree of freedom. We refer to the protocol of changing the state sum, which reduces the sum over the Hilbert space $\mathcal{H}$ down to a much smaller subset of states, Distill $[\mathcal{H}]$, as quantum distillation. The origin of this idea in two-dimensional QFTs can be traced to [4-7]. The spectral miracles in adjoint QCD in four dimensions [8-10] can also be interpreted in this language. 


\section{QUANTUM DISTILLATION IN QCD(F/ADJ)}

Consider QCD with $N_{f}=N_{c}$ flavors of fundamental massless Dirac fermions $\psi^{a}$ and one adjoint massless Weyl fermion $\lambda$ with Euclidean Lagrangian,

$$
\mathcal{L}=\frac{1}{2 g^{2}} \operatorname{tr} F_{\mu \nu}^{2}+\sum_{a=1}^{N_{f}} \bar{\psi}_{a} \gamma_{\mu} D_{\mu} \psi^{a}+2 \operatorname{tr} \bar{\lambda} \bar{\sigma}_{\mu} D_{\mu} \lambda .
$$

The global symmetry of the theory $\mathbf{G}_{\text {non-ab }}$ that acts faithfully on the Hilbert space is

$$
\frac{S U\left(N_{f}\right)_{L} \times S U\left(N_{f}\right)_{R} \times U(1)_{V} \times U(1)_{A_{D}} \times \mathbb{Z}_{2 \operatorname{gcd}\left(N_{c}, N_{f}\right)},}{\mathbb{Z}_{N_{c}} \times\left(\mathbb{Z}_{N_{f}}\right)_{L} \times\left(\mathbb{Z}_{N_{f}}\right)_{R} \times\left(\mathbb{Z}_{2}\right)_{\psi}}
$$

where $U(1)_{A_{D}}$ is the diagonal subgroup of the two classical chiral $U(1)_{A}$ acting on $\psi^{a}$ and $\lambda$, respectively. $\mathbb{Z}_{2 \operatorname{gcd}\left(N_{c}, N_{f}\right)}$ is the discrete remnant of classical chiral symmetry. By turning on a mass $m_{\lambda}>0$ for adjoint fermion, the theory can be reduced to QCD at low energy. There are two reasonable scenarios for the chiral symmetry breaking. (i) The whole axial part is broken as in QCD. (ii) All but $U(1)_{A_{D}}$ axial symmetry is broken as in $N_{f}=N_{c}$ SQCD $[11,12]$. Of course, once a mass term $m_{\lambda}$ is turned on (which is our interest in this work), $U(1)_{A_{D}}$ is lost and there is no difference between these two scenarios.

We would like to study the dynamics of the theory by using a generalized partition function

$$
\mathcal{Z}\left(\beta, \epsilon_{a}\right)=\operatorname{tr}\left[e^{-\beta H}(-1)^{F} \prod_{a=1}^{N_{f}} e^{i \epsilon_{a} Q_{a}}\right],
$$

where $Q_{a}$ are the charges corresponding to Cartan generators of $U\left(N_{f}\right)_{V}$ and $(-1)^{F}$ is grading by fermion number of $\lambda$. These operators commute with the Hamiltonian $H$ and do not alter the Hilbert space $\mathcal{H}$. In path integral formulation, this corresponds to the boundary conditions

$$
\begin{aligned}
\lambda(\beta) & =+\lambda(0), \quad \psi(\beta)=-\psi(0) \bar{\Omega}_{F}, \\
\Omega_{F} & =\operatorname{diag}\left(e^{i \epsilon_{1}}, \ldots, e^{i \epsilon_{N_{f}}}\right),
\end{aligned}
$$

where $\Omega_{F}$ is a flavor twist. $\Omega_{F}$ may also be viewed as an imaginary chemical potential, and Hilbert space grading and sum provide a physical interpretation for it.

In the presence of fundamental fermions, it is well known that the one-form (and zero form in compactified theory) center symmetry is explicitly broken, and Wilson loops (Polyakov loops for zero form) are no longer good order parameters; see [13-15]. However, consider a $U\left(N_{f}\right)_{V}$ flavor-twisted boundary condition on fermions, setting $N_{f}=N_{c}$,

$$
\Omega_{F}^{0}=\operatorname{diag}\left(1, \omega, \ldots, \omega^{N_{f}-1}\right), \quad \omega=e^{2 \pi i / N_{f}},
$$

where $\psi\left(x_{4}+\beta\right)=-\psi\left(x_{4}\right) \bar{\Omega}_{F}^{0}$. Under a gauge transformation periodic up to an element of the center, the boundary condition maps into $\psi\left(x_{4}+\beta\right)=-\psi\left(x_{4}\right) \bar{\Omega}_{F}^{0} \omega$. Since this boundary condition is different from the original one, this is a noninvariance of the partition function and explicitly breaks zero-form center symmetry. On the other hand, $\omega \bar{\Omega}_{F}^{0}$ is a cyclic permutation of $\bar{\Omega}_{F}^{0}$ and can be brought to the original boundary conditions by using cyclic permutation matrix $(S)_{a, b}=\delta_{a+1, b} \in \Gamma_{S} \subset S U\left(N_{f}\right)_{V}$, and turn the combined operation into a genuine global symmetry.

For general $N_{f}, N_{c}$, the compactified theory possesses an exact $\mathbb{Z}_{\operatorname{gcd}\left(N_{f}, N_{c}\right)}$ zero-form color-flavor center (CFC) symmetry under which Polyakov loops are charged, despite the fact that it does not possess a one-form center symmetry under which Wilson loops are charged [16-18]. We will see manifestation of this fact explicitly in holonomy potential. Therefore, we can examine the phase structure of these theories according to the CFC symmetry and pose questions about analyticity of graded partition function as a function of $\beta$. The CFC is one of the major players in our construction.

The price one pays for keeping an exact zero-form center symmetry is the reduction of the non-Abelian chiral symmetry to a subgroup which commutes with $\Omega_{F}^{0}$, the maximal Abelian subgroup $\mathbf{G}_{\max -\mathrm{ab}}$,

$$
\frac{U(1)_{L}^{N_{f}-1} \times U(1)_{R}^{N_{f}-1} \times U(1)_{V} \times U(1)_{A_{D}} \times \mathbb{Z}_{2 \operatorname{gcd}\left(N_{c}, N_{f}\right)} .}{\mathbb{Z}_{N_{c}} \times\left(\mathbb{Z}_{N_{f}}\right)_{L} \times\left(\mathbb{Z}_{N_{f}}\right)_{R} \times\left(\mathbb{Z}_{2}\right)_{\psi}}
$$

This is the exact symmetry at any finite $\beta$. When $\beta$ is much larger than the strong length scale, (2) should be viewed as approximate symmetry and ultimately recovered at $\beta \rightarrow \infty$ limit.

\section{GAUGE-HOLONOMY POTENTIALS WITHOUT AND WITH FLAVOR HOLONOMY TWISTS}

In the thermal case, as $\beta \rightarrow 0$, the theory moves to a chirally restored phase. This regime is sometimes called deconfined, but strictly speaking, center symmetry is not present due to the fundamental matter fields with thermal boundary conditions. In the small- $\beta$ regime, the potential for gauge holonomy $\Omega=e^{i \oint a_{4} d x_{4}}$ is well known [13,19],

$$
\begin{aligned}
V_{\text {1-loop,th }}(\Omega)= & \frac{2}{\pi^{2} \beta^{4}}\left\{\sum_{n=1}^{\infty} \frac{1}{n^{4}}\left[-1+(-1)^{n}\right]\left|\operatorname{tr}\left(\Omega^{n}\right)\right|^{2}\right. \\
& \left.+N_{f} \sum_{n=1}^{\infty} \frac{(-1)^{n}}{n^{4}}\left[\operatorname{tr}\left(\Omega^{n}\right)+\text { c.c. }\right]\right\},
\end{aligned}
$$

where the contributions are, respectively, from $a_{\mu}, \lambda, \psi_{a}$. 
The leading order free energy, which is found by minimizing this potential, is given by (see, e.g., $[14,15,20]$ )

$\mathcal{F}_{\text {th }}(\beta)=-\frac{\pi^{2}}{90} \frac{V_{3}}{\beta^{4}}[\underbrace{2\left(N_{c}^{2}-1\right)}_{\text {gluons }}+\frac{7}{8}(\underbrace{2\left(N_{c}^{2}-1\right)}_{\text {adj Weyl }}+\underbrace{4 N_{f} N_{c}}_{\text {fund D }})]$,

which can be interpreted in two different ways following quark-hadron duality [21,22]. The standard (microscopic) interpretation is the usual Stefan-Boltzmann free energy of asymptotically free quarks and gluons. The macroscopic interpretation is in terms of the hadronic density of states in the Hilbert space $\mathcal{H}$. The inverse Laplace transform of the partition function (for $N_{f} \sim N_{c}$ ) $\mathcal{Z}(\beta) \sim e^{-\beta \mathcal{F}_{\text {th }}} \sim e^{a N_{c}^{2} V_{3} / \beta^{3}}$ ( $a$ is a pure number) is just the density of states of hadrons in the spectrum, $\rho_{\mathrm{SB}}(E) \sim e^{E^{3 / 4} N_{c}^{1 / 2}\left(a V_{3}\right)^{1 / 4}}$, where SB stands for the Stefan-Boltzmann growth. The idea of quantum distillation is to create sufficient destructive interference in the state sum such that an effective density of states (the one associated with Distill[ $[\mathcal{H}]$ ) is not strong enough to change the phase of the theory as $\beta$ is reduced.

The gap between the minimum and maximum of the holonomy potential (7) is $\Delta V \sim O\left(N_{c}^{2}\right)$. The configuration $\Omega=\mathbb{1}_{N_{c}}$ is the minimum and governs the properties of the thermal equilibrium state. In particular, the centersymmetric configurations are close to maxima. All three terms in the potential (7) need to be defeated in order for the quantum distillation idea to work.

The effect of grading in state sum (3) maps into nondynamical flavor holonomy dependence in the gaugeholonomy potential. At one-loop order, we find

$$
\begin{aligned}
V_{1-\text { loop }, \Omega_{F}} & =V_{1-\text { loop }}^{\text {gauge }}+V_{1-\text { loop }}^{\lambda}+V_{1-\text { loop }, \Omega_{F}}^{\psi}, \\
V_{1-\text { loop }}^{\text {gauge }}+V_{1-\text { loop }}^{\lambda} & =(-1+1) \frac{2}{\pi^{2} \beta^{4}} \sum_{n=1}^{\infty} \frac{1}{n^{4}}\left|\operatorname{tr}\left(\Omega^{n}\right)\right|^{2}=0, \\
V_{1-\text { loop }, \Omega_{F}}^{\psi} & =\frac{2}{\pi^{2} \beta^{4}} \sum_{n=1}^{\infty} \frac{(-1)^{n}}{n^{4}}\left[\operatorname{tr}\left(\bar{\Omega}_{F}^{n}\right) \operatorname{tr}\left(\Omega^{n}\right)+\text { c.c. }\right] .
\end{aligned}
$$

The effect of gauge fluctuations is undone by one massless adjoint fermion (but recall that we will make adjoint fermion heavy and reduce the low energy theory to QCD.) This is true to all orders in perturbation theory, because the subclass of Feynman diagrams (in loop expansion) composed solely of $a_{\mu}$ and $\lambda$ is identical to $\mathcal{N}=1 \mathrm{SYM}$; hence, a potential cannot be generated from this class. Furthermore, at one- and two-loop order, $\lambda$ and $\psi^{a}$ are decoupled in loop expansion. Starting at three-loop order, there are diagrams involving both fundamental and adjoint fermions. However, the CFC realization can be determined reliably with two-loop knowledge.
For $\Omega_{F}=\Omega_{F}^{0}$ given in (5), since $\operatorname{tr}\left(\Omega_{F}^{0}\right)^{n}=0$ unless $n=N_{f} k, k \in \mathbb{Z}^{+}$, a number of remarkable effects take place. (i) We observe the manifestation of the exact CFC symmetry at one-loop order. The potential becomes invariant under $\mathbb{Z}_{\operatorname{gcd}\left(N_{f}, N_{c}\right)}$ symmetry since all terms of the form $\operatorname{tr}\left(\Omega^{n}\right), n \neq N_{f} k$ vanish. (ii) The gap between the minimum and maximum of the potential, for $N_{f} \sim N_{c}$, becomes $\Delta V_{1 \text {-loop, } \Omega_{F}^{0}} \sim O\left(1 / N_{c}^{2}\right)$, i.e., the one-loop potential is extremely frustrated and it collapses. At one-loop order, one obtains an exponentially increasing number of degenerate minima, $\mathfrak{N}_{\min }\left(N_{c}\right) \approx \frac{2^{2 N_{c}-1} N_{c}^{-3 / 2}}{\sqrt{\pi}}$. In the $N_{c} \rightarrow \infty$ limit, the gauge-holonomy potential vanishes at one-loop order and the set of minima becomes continuous similar to supersymmetric theories. (iii) The graded free energy at the level of one-loop analysis (for $N_{f} \sim N_{c}$ ) takes the form

$$
\begin{aligned}
\mathcal{F}_{\Omega_{F}^{0}}(\beta) & =-\frac{\pi^{2}}{90} \frac{V_{3}}{\beta^{4}}[\underbrace{2\left(N_{c}^{2}-1\right)}_{\text {gluons }}-\underbrace{2\left(N_{c}^{2}-1\right)}_{\text {adj Weyl }}+\underbrace{\frac{7}{2} \frac{1}{N_{c}^{2}}}_{\text {fund D }}] \\
& =-\frac{\pi^{2}}{90} \frac{V_{3}}{\beta^{4}}\left[\frac{7}{2} \frac{1}{N_{c}^{2}}\right] \rightarrow 0, \quad N_{c} \rightarrow \infty .
\end{aligned}
$$

This is the effect of quantum distillation. It is as if there is merely $\frac{1}{N_{c}^{2}}$ quark degree of freedom in the system instead of $\sim N_{c}^{2}$ bosonic and $\sim N_{c}^{2}$ fermionic degrees of freedom. The corresponding scaling of the graded partition function is $\mathcal{Z}(\beta) \sim e^{\frac{a}{N_{c}^{2}} V_{3} / \beta^{3}}$, and the effective density of states of the hadronic states in $\operatorname{Distill}[\mathcal{H}]$ is given by $\rho_{\Omega_{F}^{0}}(E) \sim e^{\frac{1}{N_{c}^{1 / 2}} E^{3 / 4}\left(a V_{3}\right)^{1 / 4}}$.

In order to determine CFC realization, we need a twoloop result in the presence of the grading. To this end, we generalize earlier thermal studies of Refs. [23,24,25] to incorporate flavor holonomy. We use Eq. (17) in [24] and Eq. (5.11) in [25]. These two results at first sight look different, but due to nontrivial Bernoulli polynomial identities, they are actually the same.

The two-loop potential in the presence of the flavortwisted boundary conditions is (4)

$$
\begin{aligned}
& V_{2-1, \Omega_{F}}^{\psi} \\
& =\frac{g^{2}}{\beta^{4}} \frac{3}{\pi^{4}}\left\{-\frac{N_{c}^{2}-1}{8 N_{c}} \sum_{n=1}^{\infty} \frac{(-1)^{n}}{n^{4}}\left[\operatorname{Tr}\left(\bar{\Omega}_{F}^{n}\right) \operatorname{Tr}\left(\Omega^{n}\right)+\text { c.c. }\right]\right. \\
& \left.\quad+\frac{N_{f}}{24} \sum_{n=1}^{\infty} \frac{\left|\operatorname{Tr}\left(\Omega^{n}\right)\right|^{2}}{n^{4}}\right\} .
\end{aligned}
$$

Again, in the $\Omega_{F}=\Omega_{F}^{0}$ background, in the first sum, all but $n=N_{f} k$ terms vanish, and the sum is $O\left(\left(g^{2} N_{c}\right) / N_{c}^{2}\right)$ without altering any of the results of one-loop analysis. In the large- $N_{c}$ Veneziano limit [26] of $\mathrm{QCD}(\mathrm{F} / \mathrm{adj})$, the combined one- and two-loop potential takes a simple and beautiful form 


$$
V_{1-1, \Omega_{F}^{0}}+V_{2-1, \Omega_{F}^{0}}=+\frac{g^{2} N_{f}}{8 \pi^{4} \beta^{4}} \sum_{n=1}^{\infty} \frac{\left|\operatorname{Tr}\left(\Omega^{n}\right)\right|^{2}}{n^{4}} .
$$

This is one of the main results of this work. Fundamental fermions with $\Omega_{F}^{0}$-twisted boundary conditions at two-loop order is capable of stabilizing the CFC symmetry, by inducing center stabilizing double-trace terms at two-loop order. In this sense, they behave similar to adjoint fermions with periodic boundary conditions [27]. The minimum of the gauge-holonomy potential is now at

$$
\left.\Omega\right|_{\min }=\omega^{-\left(N_{c}-1\right) / 2} \operatorname{diag}\left(1, \omega, \ldots, \omega^{N_{c}-1}\right) .
$$

The stability of center symmetry is robust to all loop orders in perturbation theory and nonperturbatively in the weak coupling small $-\beta$ regime. The stability is the image of the quantum distillation over the Hilbert space in the gaugeholonomy potential.

Turning on $m_{\lambda}$, the balance between the gauge fluctuation and adjoint fermion breaks in favor of CFC breaking. However, since the two-loop effects of the fundamental fermions is also CFC stabilizing, the full $\mathbb{Z}_{N_{c}}$ center symmetry can be kept intact provided $m_{\lambda} \leq m_{\lambda}^{*}=\frac{\left(g^{2} N_{c}\right)^{1 / 2}}{N_{c} \beta \pi}$. Therefore, provided $\Lambda \ll m_{\lambda} \leq m_{\lambda}^{*}$ (which is easily achieved at finite $N_{c}$ ), the IR theory is essentially QCD(F) both on $\mathbb{R}^{4}$ and on $\mathbb{R}^{3} \times S^{1}$. In other words, in this window, the adjoint fermion does not decouple from the holonomy potential, but it does decouple from other aspects of the long distance physics, such as chiral Lagrangian.

Therefore, the net effect of quantum distillation is the $1 / N_{c}^{4}$ suppression in free energy (at least at two-loop order) and the suppression of the hadronic density of states

$$
\begin{aligned}
\rho_{\mathrm{SB}}(E) & \sim e^{E^{3 / 4} N_{c}^{1 / 2}\left(a\left(g^{2} N_{c}\right) V_{3}\right)^{1 / 4}} \\
& \mapsto \rho_{\Omega_{F}^{0}}(E) \sim e^{\frac{1}{N_{c}^{1 / 2}} E^{3 / 4}\left(a\left(g^{2} N_{c}\right) V_{3}\right)^{1 / 4}},
\end{aligned}
$$

where $a\left(g^{2} N_{c}\right)$ is a series expansion in 't Hooft coupling. It is highly plausible, similar to [10], that the growth $\exp \left(V_{3}^{1 / 4} E^{3 / 4}\right)$ expected in a standard local 4D theory in spatial volume $V_{3}$ cancels and $\rho_{\Omega_{F}^{0}}(E)$ turns out to have a scaling associated with a two-dimensional QFT in the $N_{c} \rightarrow \infty$ limit.

In the small circle regime where CFC remains unbroken, the chiral symmetry breaks spontaneously by the condensation of monopole flux operators as described in [28]. In the Appendix, we present this mechanism in operator formalism in contrast with [28]. Therefore, we conjecture that the small- $S^{1}$ chirally broken phase of $\mathrm{QCD}(\mathrm{F} / \mathrm{adj})$ with $\Lambda \ll m_{\lambda}<m_{\lambda}^{*}$ is adiabatically connected to the strong coupling regime, and quantum distillation achieves its goal, the continuity of the partition function. We cannot prove this conjecture, but instead we can prove weaker statement that the vacuum structure of the theory on any size $\mathbb{R}^{3} \times S^{1}$ and $\mathbb{R}^{4}$ is controlled by the same mixed 't Hooft anomaly condition.

\section{MIXED ANOMALY AND PERSISTENT ORDER}

On $\mathbb{R}^{4}$, the faithful symmetry of the $\Lambda \ll m_{\lambda}<m_{\lambda}^{*}$ theory includes $G_{1}=S U\left(N_{f}\right)_{V} / \mathbb{Z}_{\operatorname{gcd}\left(N_{f}, N_{c}\right)}$ because $\mathbb{Z}_{\operatorname{gcd}\left(N_{f}, N_{c}\right)} \subset \mathbb{Z}_{N_{c}}$ is part of gauge structure, and therefore, is not a symmetry. The theory also has a $G_{2}=\mathbb{Z}_{2 N_{f}}$ chiral symmetry, which resides in continuous chiral symmetry. By using the techniques of [29-34], it is possible to show that there is a mixed anomaly between these two symmetries.

Introducing the $S U\left(N_{f}\right)$ gauge field $A$, the theory becomes $S U\left(N_{f}\right) \times S U\left(N_{c}\right)$ quiver theory (bifundamental QCD), which has a genuine $\mathbb{Z}_{\operatorname{gcd}\left(N_{f}, N_{c}\right)}$ one-form symmetry. To gauge the correct symmetry, $G_{1}$, the one-form symmetry must also be gauged, and for this purpose, we introduce a two-form gauge field $B$. In quiver theory, only $\mathbb{Z}_{2 \operatorname{gcd}\left(N_{f}, N_{c}\right)} \subset \mathbb{Z}_{2 N_{f}}$ chiral symmetry is present. Generalizing Ref. [32] to arbitrary $N_{f}$ and $N_{c}$, we observe that under the discrete $\chi \mathrm{S}$ transformation $h$, the partition function fails to be invariant,

$$
\mathcal{Z}(h(A, B))=e^{-i \frac{2 \operatorname{lem}\left(N_{f}, N_{c}\right)}{4 \pi}} \int B \wedge B \mathcal{Z}(A, B),
$$

provided

$$
\frac{2 \operatorname{lcm}\left(N_{f}, N_{c}\right)}{\left(\operatorname{gcd}\left(N_{f}, N_{c}\right)\right)^{2}} \in \mathbb{Q} \backslash \mathbb{Z} .
$$

For all $N_{f}=N_{c} \geq 3$, there is a mixed anomaly since $\frac{2}{N_{c}} \in \mathbb{Q} \backslash \mathbb{Z}$ in agreement with [32].

This mixed 't Hooft anomaly implies that a unique, gapped ground state is impossible. Therefore, either (i) $S U\left(N_{f}\right)_{V} / \mathbb{Z}_{\operatorname{gcd}\left(N_{f}, N_{c}\right)}$ or (ii) $\mathbb{Z}_{2 N_{f}}$ or (iii) both are spontaneously broken, or (iv) IR theory has to be a CFT. In the present case, we can rule out (i) and (iii) because of the Vafa-Witten theorem, which asserts that in vectorlike theories, vectorlike global symmetries cannot be spontaneously broken as long as one assures positivity of the measure [35]. Fortunately, the grading (3) respects this positivity. For QCD-like theories, both $\chi \mathrm{SB}$ and CFT options are equally reasonable. From now on, we work with the assumption that $\mathbb{Z}_{2 N_{f}}$ discrete symmetry is broken. As observed in [36], there exists no order parameter which is charged under the discrete $\chi \mathrm{S}$ but not under continuous $\chi \mathrm{S}$ (the opposite statement is not true), implying spontaneous breaking of $S U\left(N_{f}\right)_{A}$. Therefore, massless pions must exist in the spectrum.

Reference [30] recently showed that a mixed anomaly between a one-form symmetry and zero-form symmetry 
persists upon compactification on $\mathbb{R}^{3} \times S^{1}$; see also $[33,34]$. This is in sharp contrast with mixed anomalies involving only zero-form symmetries originally discussed by 't Hooft [29] which do not survive compactification. However, there is an important exception for the latter [32]. Assume we have zero-form symmetries $G_{1} \times G_{2}$, where $G_{1}=\tilde{G}_{1} / \Gamma$ and gauging $\tilde{G}_{1}$ turns $\Gamma$ into a genuine oneform symmetry. This is precisely the case in $\mathrm{QCD}(\mathrm{F} / \mathrm{adj})$. Then, a triple-mixed anomaly persists upon compactification provided some conditions are satisfied.

The anomaly (15) persists compactification on $\mathbb{R}^{3} \times S^{1}$ if and only if one uses a flavor-twisted boundary condition by $\Omega_{F}^{0}$ [37]. Indeed, these boundary conditions are used in [4-7] to preserve adiabatic continuity. In QCD(F/adj), the combination of center transformation and $\Gamma_{S}$ cyclic permutation subgroup of $S U\left(N_{f}\right)_{V}$ remains as a true zero-form symmetry of the compactified theory. This is identified as CFC symmetry in [16]. Introducing the background gauge field for $U(1)^{N_{f}-1}, \mathbb{Z}_{\operatorname{gcd}\left(N_{f}, N_{c}\right)}$ emerges as a one-form symmetry. We also introduce a two-form field $B^{(2)}$ and one-form field $B^{(1)}$ associated with one-form and zero-form part of center symmetry $\mathbb{Z}_{\operatorname{gcd}\left(N_{f}, N_{c}\right)}$. Then, the partition function fails to be invariant under $h \in \mathbb{Z}_{2 N_{f}}$ transformation as

$$
\begin{aligned}
\mathcal{Z}_{\Omega_{F}^{0}} & \left(h\left(A_{K}, B^{(2)}, B^{(1)}\right)\right) \\
\quad & e^{-i \frac{2 \operatorname{lem}\left(N_{f}, N_{c}\right)}{2 \pi} \int B^{(2)} \wedge B^{(1)}} \mathcal{Z}_{\Omega_{F}^{0}}\left(A_{K}, B^{(2)}, B^{(1)}\right) .
\end{aligned}
$$

Therefore, there is a triple mixed anomaly between $\Gamma_{S}$ shift symmetry, Abelianized flavor symmetry $U(1)^{N_{f}-1} /$ $\mathbb{Z}_{\operatorname{gcd}\left(N_{f}, N_{c}\right)}$, and the discrete $\chi \mathrm{S} \mathbb{Z}_{2 N_{f}} \subset U(1)_{A}^{N_{f}-1}$ provided (16) holds, the same condition as in $\mathbb{R}^{4}$. Indeed, this anomaly polynomial naturally descends from the one on $\mathbb{R}^{4}(15)$ with the substitution $B=B^{(2)}+B^{(1)} \wedge \beta^{-1} d x^{4}$. Again, the mixed anomaly implies that the ground state cannot be unique and gapped. The options are $U(1)_{A}^{N_{f}-1}$ chiral symmetry breaking, CFC breaking, spontaneous breaking of both, or a CFT behavior at low energy. Semiclassics (described in the Appendix) proves that the first option takes place with the use of $\Omega_{F}^{0}$ twisting at small $\beta$.

\section{CONCLUSION}

Despite the fact that we did not prove adiabatic continuity between the weak and strong coupling regimes, we reduced the possibilities to just a few thanks to persistent order due to the mixed anomaly.

On small $\mathbb{R}^{3} \times S^{1}$, we showed that one can keep the CFC symmetry intact with a unique choice of boundary conditions in $\mathrm{QCD}$ (F/adj). One can sufficiently decouple adjoint fermions to reduce the theory to real $\mathrm{QCD}(\mathrm{F})$. The preservation of the CFC symmetry is a manifestation of the distillation of Hilbert space, similar to what $\operatorname{tr}\left[e^{-\beta H}(-1)^{F}\right]$ achieves in supersymmetric theories.

We showed that the twisted boundary conditions which satisfy maximal quantum distillation is the one for which the anomaly polynomial on $\mathbb{R}^{4}$ naturally descends to $\mathbb{R}^{3} \times S^{1}$. On small $S^{1}$, we proved chiral symmetry breaking rigorously with semiclassical methods. On large $S^{1}$, and $\mathbb{R}^{4}$, the ground states are controlled by the same mixed anomaly structure as small $S^{1}$.

Our work has interesting connections with spectral conspiracies in non-SUSY theories [10,38,39], and volume independence [8,40-43] whose working examples must have an interpretation in terms of quantum distillation.

Our construction creates a sign problem in the Hamiltonian formalism, in the state sum, but not in the Euclidean path integral formulation. In this sense, it is a good sign "problem," opposite to the notorious QCD sign problem at finite chemical potential. Therefore, it is possible to test our analytic construction via numerical lattice simulations involving light fermions.

\section{ACKNOWLEDGMENTS}

We are grateful to Aleksey Cherman, David Gross, and Yuya Tanizaki for discussions. M. U. acknowledges support from the U.S. Department of Energy, Office of Science, Office of Nuclear Physics under Award No. DE-FG02-03ER41260.

\section{APPENDIX: CHIRAL SYMMETRY BREAKING}

In this short appendix, we present an operator description of chiral symmetry breaking on small $\mathbb{R}^{3} \times S^{1}$ in the regime where color-flavor center symmetry is unbroken and $\Lambda \ll m_{\lambda}<m_{\lambda}^{*}$. We set $N_{f}=N_{c}$ for simplicity. Our result is same as [28], translated to operator language, and has the virtue of providing interesting insights.

At the gauge-holonomy configuration (13), the theory dynamically Abelianizes down to $U(1)^{N_{c}-1}$ at distances larger than inverse $W$-boson mass. The $U(1)^{N_{c}-1}$ photons can be dualized to gapless scalars, $F \sim * d \sigma$, which remain gapless to all orders in perturbation theory. The gaplessness is protected by the topological shift symmetry,

$$
\left[U(1)_{J}\right]^{N_{c}-1}: \sigma \rightarrow \sigma+\varepsilon, \quad \mathcal{J}_{\mu}=\partial_{\mu} \sigma .
$$

Nonperturbatively, there are monopole-instanton effects, which come in $N_{c}$ types, associated with the affine root system of $\mathfrak{g} \mathfrak{t}\left(N_{c}\right)$ Lie algebra [44,45]. The form of the monopole operators depend on the interplay between the flavor holonomy and gauge holonomy. Without loss of generality, and with a particular choice of flavor holonomy, the monopole operators can be written as

$$
\mathcal{M}_{i} \sim e^{-S_{i}} e^{-\frac{4 \pi}{g^{2}} \alpha_{i} \cdot \phi+i \alpha_{i} \cdot \sigma}\left(\psi_{L}^{i} \psi_{R i}\right) .
$$


Consider a collection of $n_{i}$ monopoles of type- $i$ $i=1, \ldots, N_{c}$ sprinkled in between two asymptotic time slice. Then, the magnetic charge nonconservation is

$$
\begin{aligned}
\Delta \mathbf{Q}_{m} & \equiv \mathbf{Q}_{m}(t=\infty)-\mathbf{Q}_{m}(t=-\infty) \\
& =\left.\int d^{2} x F_{12}\right|_{t=-\infty} ^{t=+\infty}=\int_{S_{\infty}^{2}} F_{12} \\
& =\frac{4 \pi}{g} \sum_{i=1}^{N_{c}} n_{i} \alpha_{i} .
\end{aligned}
$$

These charges violate emergent $\left[U(1)_{J}\right]^{N_{c}-1}$ explicitly. Based on this violation, which is same as in the old Polyakov model [46], one may be tempted to think that the $\sigma$ fluctuations will be nonperturbatively gapped due to proliferation of monopoles. But the story is actually opposite. In this background, the axial charge associated with $\mathbf{G}_{\max -a b}(6)$ is also not conserved,

$$
\Delta \mathbf{Q}^{5}=\sum_{i=1}^{N_{f}} n_{i} \alpha_{i} .
$$

Naively, this would mean that $\mathbf{G}_{\max -\mathrm{ab}}$ is anomalous. But this is impossible since it lives in $\mathbf{G}_{\mathbf{n o n}-\mathbf{a b}}$, which is manifestly anomaly free. The resolution of this puzzle is generalization of a mechanism discovered by Affleck et al.
[47] in a gauge theory on $\mathbb{R}^{3}$. A linear combination of these two charges is nonperturbatively conserved,

$$
\Delta \tilde{\mathbf{Q}} \equiv \Delta\left(\frac{g}{4 \pi} \mathbf{Q}_{m}-\mathbf{Q}^{5}\right)=0
$$

Since the $\mathbf{G}_{\mathbf{a b}}$ is the true microscopic symmetry, and $\left[U(1)_{J}\right]^{N_{c}-1}$ is emergent symmetry to all orders in perturbation theory, this mechanism is present so that the chiral charge of the fermion bilinear is transferred into gauge fluctuations. In the IR theory, gauge fluctuations (dual photon field) become chirally charged.

The pure flux part of the monopole operators can be combined into a diagonal component of the matrix field, which can be interpreted as the chiral field of the chiral Lagrangian $\Sigma(x)=\operatorname{Diag}\left(e^{i \alpha_{1} \cdot \sigma}, \ldots, e^{i \alpha_{N_{c}} \cdot \sigma}\right) . \Sigma$ manifold is the maximal torus of chiral symmetry, and (A5) forbids formation of a potential on it. Choosing a point on the $\Sigma$ field manifold corresponds to spontaneous chiral symmetry breaking, and IR theory is described by $S=\int_{\mathbb{R}^{3} \times S^{1}} \frac{f_{\pi}^{2}}{4} \operatorname{tr}\left|\partial_{\mu} \Sigma\right|^{2}$. Turning on a small mass for quarks breaks chiral symmetry softly and induces a potential $\sim m_{\psi} e^{-S_{0}} \operatorname{tr}\left(\Sigma+\Sigma^{\dagger}\right)$. The theory acquires a nonperturbatively induced mass gap on small $\mathbb{R}^{3} \times S^{1}$. For details of this chiral symmetry breaking mechanism in path integral description, see [28].
[1] E. Witten, Constraints on supersymmetry breaking, Nucl. Phys. B202, 253 (1982).

[2] M. Shifman and M. Unsal, QCD-like theories on R(3) x S(1): A smooth journey from small to large $\mathrm{r}(\mathrm{S}(1))$ with doubletrace deformations, Phys. Rev. D 78, 065004 (2008).

[3] M. Unsal and L. G. Yaffe, Center-stabilized Yang-Mills theory: Confinement and large $N$ volume independence, Phys. Rev. D 78, 065035 (2008).

[4] G. V. Dunne and M. Unsal, Resurgence and trans-series in quantum field theory: The $\mathrm{CP}(\mathrm{N}-1)$ model, J. High Energy Phys. 11 (2012) 170.

[5] A. Cherman, D. Dorigoni, and M. Unsal, Decoding perturbation theory using resurgence: Stokes phenomena, new saddle points and Lefschetz thimbles, J. High Energy Phys. 10 (2015) 056.

[6] T. Sulejmanpasic, Global Symmetries, Volume Independence, and Continuity in Quantum Field Theories, Phys. Rev. Lett. 118, 011601 (2017).

[7] G. V. Dunne, Y. Tanizaki, and M. Ünsal, Quantum distillation of Hilbert spaces, semi-classics and anomaly matching, J. High Energy Phys. 08 (2018) 068.

[8] G. Basar, A. Cherman, D. Dorigoni, and M. Unsal, Large N Volume Independence and an Emergent Fermionic Symmetry, Phys. Rev. Lett. 111, 121601 (2013).
[9] G. Basar, A. Cherman, and D. A. McGady, Bose-Fermi degeneracies in large $N$ adjoint QCD, J. High Energy Phys. 07 (2015) 016.

[10] A. Cherman, M. Shifman, and M. Ünsal, Bose-Fermi cancellations without supersymmetry, Phys. Rev. D 99, 105001 (2019).

[11] I. Affleck, M. Dine, and N. Seiberg, Dynamical supersymmetry breaking in supersymmetric QCD, Nucl. Phys. B241, 493 (1984).

[12] O. Aharony, J. Sonnenschein, M. E. Peskin, and S. Yankielowicz, Exotic nonsupersymmetric gauge dynamics from supersymmetric QCD, Phys. Rev. D 52, 6157 (1995).

[13] D. J. Gross, R. D. Pisarski, and L. G. Yaffe, QCD and instantons at finite temperature, Rev. Mod. Phys. 53, 43 (1981).

[14] J. I. Kapusta and C. Gale, Finite-Temperature Field Theory: Principles and Applications, Cambridge Monographs on Mathematical Physics (Cambridge University Press, Cambridge, United Kingdom, 2011).

[15] M. Laine and A. Vuorinen, Basics of thermal field theory, Lect. Notes Phys. 925, 1 (2016).

[16] A. Cherman, S. Sen, M. Unsal, M. L. Wagman, and L. G. Yaffe, Order Parameters and Color-Flavor Center Symmetry in QCD, Phys. Rev. Lett. 119, 222001 (2017). 
[17] T. Iritani, E. Itou, and T. Misumi, Lattice study on QCD-like theory with exact center symmetry, J. High Energy Phys. 11 (2015) 159.

[18] E. Poppitz and T. Sulejmanpasic, (S)QCD on $\mathbb{R}^{3} \times \mathbb{S}^{1}$ : Screening of Polyakov loop by fundamental quarks and the demise of semi-classics, J. High Energy Phys. 09 (2013) 128.

[19] N. Weiss, The Wilson line in finite temperature gauge theories, Phys. Rev. D 25, 2667 (1982).

[20] O. Philipsen, The QCD equation of state from the lattice, Prog. Part. Nucl. Phys. 70, 55 (2013).

[21] M. A. Shifman, Quark hadron duality, in At the Frontier of Particle Physics. Handbook of QCD Vols. 1-3 (World Scientific, Singapore, 2001), pp. 1447-1494.

[22] M. Shifman, Highly excited hadrons in QCD and beyond, in Quark-Hadron Duality and the Transition to PQCD (World Scientific, Singapore, 2005), pp. 171-191, https://doi.org/ 10.1142/9789812774132_0025.

[23] C. P. Korthals Altes, Constrained effective potential in hot QCD, Nucl. Phys. B420, 637 (1994).

[24] C. P. Korthals Altes, R. D. Pisarski, and A. Sinkovics, The potential for the phase of the Wilson line at nonzero quark density, Phys. Rev. D 61, 056007 (2000).

[25] Y. Guo and Q. Du, Two-loop perturbative corrections to the constrained effective potential in thermal QCD, J. High Energy Phys. 05 (2019) 042.

[26] G. Veneziano, Some aspects of a unified approach to gauge, dual and Gribov theories, Nucl. Phys. B117, 519 (1976).

[27] P. Kovtun, M. Unsal, and L. G. Yaffe, Volume independence in large N(c) QCD-like gauge theories, J. High Energy Phys. 06 (2007) 019.

[28] A. Cherman, T. Schäfer, and M. Ünsal, Chiral Lagrangian from Duality and Monopole Operators in Compactified QCD, Phys. Rev. Lett. 117, 081601 (2016).

[29] G. 't Hooft, Naturalness, chiral symmetry, and spontaneous chiral symmetry breaking, NATO Sci. Ser. B 59, 135 (1980).

[30] D. Gaiotto, A. Kapustin, Z. Komargodski, and N. Seiberg, Theta, time reversal, and temperature, J. High Energy Phys. 05 (2017) 091.

[31] D. Gaiotto, A. Kapustin, N. Seiberg, and B. Willett, Generalized global symmetries, J. High Energy Phys. 02 (2015) 172.
[32] Y. Tanizaki, T. Misumi, and N. Sakai, Circle compactification and 't Hooft anomaly, J. High Energy Phys. 12 (2017) 056.

[33] H. Shimizu and K. Yonekura, Anomaly constraints on deconfinement and chiral phase transition, Phys. Rev. D 97, 105011 (2018).

[34] Z. Komargodski, T. Sulejmanpasic, and M. Ünsal, Walls, anomalies, and (De)confinement in quantum antiferromagnets, Phys. Rev. B 97, 054418 (2018).

[35] C. Vafa and E. Witten, Restrictions on symmetry breaking in vector-like gauge theories, Nucl. Phys. B234, 173 (1984).

[36] A. Cherman and M. Unsal, Critical behavior of gauge theories and Coulomb gases in three and four dimensions, arXiv:1711.10567.

[37] Y. Tanizaki, Y. Kikuchi, T. Misumi, and N. Sakai, Anomaly matching for the phase diagram of massless $\mathbb{Z}_{N}$-QCD, Phys. Rev. D 97, 054012 (2018).

[38] D. Kutasov and N. Seiberg, Number of degrees of freedom, density of states and tachyons in string theory and CFT, Nucl. Phys. B358, 600 (1991).

[39] K. R. Dienes, M. Moshe, and R. C. Myers, String Theory, Misaligned Supersymmetry, and the Supertrace Constraints, Phys. Rev. Lett. 74, 4767 (1995).

[40] T. Eguchi and H. Kawai, Reduction of Dynamical Degrees of Freedom in the Large N Gauge Theory, Phys. Rev. Lett. 48, 1063 (1982).

[41] A. Gonzalez-Arroyo and M. Okawa, The twisted EguchiKawai model: A reduced model for large N lattice gauge theory, Phys. Rev. D 27, 2397 (1983).

[42] A. Gonzalez-Arroyo and M. Okawa, Large $N$ reduction with the twisted Eguchi-Kawai model, J. High Energy Phys. 07 (2010) 043.

[43] M. Garcia Perez, A. Gonzalez-Arroyo, and M. Okawa, Volume independence for Yang-Mills fields on the twisted torus, Int. J. Mod. Phys. A 29, 1445001 (2014).

[44] K.-M. Lee and P. Yi, Monopoles and instantons on partially compactified D-branes, Phys. Rev. D 56, 3711 (1997).

[45] T. C. Kraan and P. van Baal, Monopole constituents inside SU(n) calorons, Phys. Lett. B 435, 389 (1998).

[46] A. M. Polyakov, Quark confinement and topology of gauge theories, Nucl. Phys. B120, 429 (1977).

[47] I. Affleck, J. A. Harvey, and E. Witten, Instantons and (Super)symmetry breaking in $(2+1)$-dimensions, Nucl. Phys. B206, 413 (1982). 